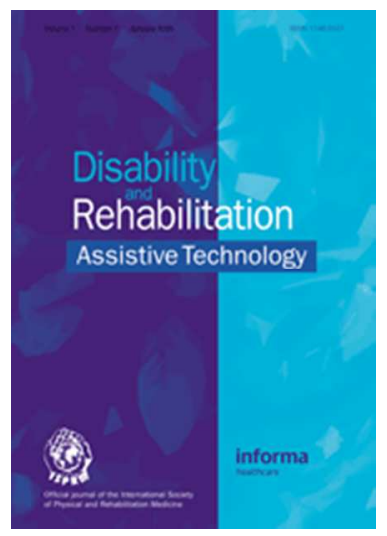

\title{
Rehabilitation professionals' perceptions of the use of new visualisation software tools with people with stroke.
}

\begin{tabular}{|r|l|}
\hline Journal: & Disability and Rehabilitation: Assistive Technology \\
\hline Manuscript ID & TIDT-03-2015-008.R1 \\
\hline Manuscript Type: & Original Research \\
\hline Keywords: & $\begin{array}{l}\text { stroke rehabilitation, biomechanical data, visual software technology, } \\
\text { qualitative research }\end{array}$ \\
\hline \multicolumn{2}{|l}{} \\
\hline
\end{tabular}

SCHOLARONE

Manuscripts 
Key words: stroke rehabilitation, biomechanical data, visual software technology, qualitative research

\begin{abstract}
Purpose - The envisage programme of research was funded to explore and evaluate the use of visualisation software tools using biomechanical data within rehabilitation. Three work packages were developed to evaluate the impact of the tools within stroke rehabilitation. The research presented here aimed to explore the perceptions of rehabilitation therapists about the use of the visualisation software tools in the context of future randomised controlled trials and stroke rehabilitation practice.

Methods - Sixteen therapists working in a range of stroke rehabilitation contexts participated in semi-structured interviews. Interview questions explored their current practice, and the perceived impact of the new visualisation technologies on their workplace environment and practice. Framework analysis was used to analyse the textual data.

Results - In general the stroke therapists were enthusiastic about the potential application of the visualisation software tools. Three themes were identified through qualitative framework analysis: potential uses of the visualisation tools; integration within current service provision; and trial involvement.

Conclusions - The study highlights important contextual considerations which may impact significantly on the success of novel technologies in stroke rehabilitation. Normalisation process theory was proposed as a useful process evaluation methodology to optimise both trial evaluation and future service implementation.
\end{abstract}




\section{Introduction}

An understanding of the biomechanics of movement disorders is an important prerequisite for successful physical rehabilitation, for example, with people with stroke [1]. However, the complexities of communicating biomechanical data to a variety of health care disciplines and service users have meant that the full potential of biomechanics to optimise rehabilitation has yet to be realised, in spite of exciting developments in the field. Previous research by the authors explored the opportunities afforded by technological visualisations to enhance understanding of both health care professionals and services users of complex biomechanical information [2]. Findings were promising, suggesting that visualisations could improve the accessibility of complex data.

The envisage programme, funded by the UK cross-research council Lifelong Health and Wellbeing (LLHW2) initiative, built on the findings of this previous research through a multidisciplinary collaboration including design specialists, biomechanists, rehabilitation professionals and service users. The aim of this programme of work was to explore the potential of visualisations of biomechanical data to positively impact on rehabilitation outcomes for service users, with a variety of different impairments in a range of contexts. Three discrete work packages (WPs) were developed to investigate the application of novel visualisation software technology with service users with stroke; lower limb rehabilitation (WP4, ISRCTN registry number: ISRCTN79005974), (WP4a, ISRCTN9005974) upper limb rehabilitation and (WP5, ISRCTN52126764) for people who had been referred for the fitting of an ankle foot orthosis (AFO) following stroke. Each work package evaluated the effect of the visualisation software tool intervention on service user outcomes in exploratory 
Phase II randomised clinical trials following the MRC Framework for developing and evaluating complex interventions [3].

The visualisation technology employed and evaluated within these trials has been described in more detail elsewhere [4], but in brief comprised the use of motion capture technologies to present the individual patient's movement as a visual mannequin on a computer screen. Information about each of the systems used is summarised below in table 1.

\section{INSERT TABLE 1 ABOUT HERE}

There is little published research evaluating the use of visualisation technology in stroke rehabilitation, although an expanding body of literature explores the impact of novel computer technologies such as augmented or virtual reality within this field. For example, de Assis et al [5], Green and Wilson [6], Mirelman et al [7], Sampson et al [8] and Yang et al [9] all report positive benefits for adults and children of using virtual reality applications within the context of both upper and lower limb rehabilitation. A recent systematic review of randomised controlled trials evaluating 'virtual reality therapy' confirms these positive findings in terms of body function and activity (employing the International Classification of Functioning, Disability and Health terminology [10]), but calls for larger trials to replicate these promising findings, to explore cost effectiveness and to include participation outcome measures [11]. 
Qualitative research methodologies have frequently been used to explore both service user and provider experience of stroke rehabilitation (eg Rosewilliam et al 2011 [12], Nicholson et al 2014 [13], Levack et al 2011 [14]), and the contribution of qualitative research methods to compliment RCT designs evaluating health interventions is increasingly being recognised and promoted [15] [16]. Recent mixed methods studies have sought to elicit user feedback to inform the design of computer-based technology in stroke rehabilitation [17] [18]. Key elements central to the successful development and utilisation of computer technology in stroke rehabilitation include an appreciation of the individualised needs of the service user and an understanding of the environmental context. However, few researchers have focused on stakeholders' understandings, expectations, and the perceived impact of novel technologies within stroke rehabilitation. One notable exception explored stroke service users and care-givers' perspectives about how computer based assistive technology influenced their daily lives [19]. The authors found that a system of computer generated personalised reminders for people living in the community with stroke and impaired cognition increased their sense of control, created daily structure and facilitated renewed social contacts, suggesting some of the ways in which these technologies might be deployed.

Normalisation Process Theory (or NPT) is a mid-range sociological theory developed to explain the social processes through which new or modified ways of thinking, enacting and organising work ('practices') become integrated within healthcare (and other organisational contexts) [20] [21]. Table 2 identifies and defines the four core constructs through which normalisation (or the routine embedding of a practice) occurs [22]. 


\title{
INSERT TABLE 2 ABOUT HERE
}

\begin{abstract}
Within the context of NPT, Murray et al (2010) [23] propose that the perspectives of health care service providers about complex health interventions and practices are an important determinant of their successful implementation within routine health care. We therefore wanted to explore the potential utility of NPT in understanding the possible challenges and facilitators to the routine use of the novel technology used within this study, as suggested in discussion about wider practices of rehabilitation professionals. The primary aim of the research reported in this paper was to capture the pre-trial perspectives and expectations of rehabilitation therapists on the potential use and role of the visualisation software tools in the context of stroke rehabilitation.
\end{abstract}

\section{Methodology}

\section{Approach and Methods}

As the three proposed trials involved NHS stroke patients and clinicians, pre-trial workshops and training sessions were set up to discuss the trial processes in more depth, allow a range of clinicians to see the equipment being tested and discuss any issues or concerns prior to the trials starting in the clinical settings. These pre-trial workshops and training sessions provided an ideal opportunity to identify clinicians who had been shown the visualisation technologies who could be approached regarding potential participation in this qualitative study. NHS and institutional ethics and governance approvals were obtained by each of the three stroke trial leads 
(WP4, West of Scotland Research Ethics Committee 4 Ref: 11/AL/0184; WP4a West of Scotland Research Ethics Committee 2 Ref: 11/AL/0260; WP5 West of Scotland Research Ethics Committee 4 Ref: 11/AL/0166).

A 'subtle realist' position was adopted within this qualitative research study, which acknowledges an underlying reality, but recognises that this is mediated through individual perceptions [24]. This ontological position has implications for the role of the researcher in qualitative research, which, in contrast to the required objectivity in experimental research, posits that data are 'co-constructed' by the researcher and research participant within the context of an interview or focus group [25]. In challenging one consensual, agreed version of reality, a subtle realist ontology also impacts on the means selected to demonstrate rigour and good scholarship within qualitative research. In contrast to positivist criteria of reliability and validity, strategies to promote 'trustworthiness' in qualitative research include peer debriefing, inclusion of rich contextual information to inform analysis ('thick description') and use of a research journal to promote reflection [24].

Semi-structured interviews were identified as an appropriate data generation method, selected for their fluid and flexible structure which permits the emergence of interviewees' situated perspectives [26]. Focus groups were also considered, but rejected as we were more interested in the in-depth views of individual rehabilitation professionals than a collective account generated through interaction between participants. In the event, both individual and group interviews were completed, the latter because of the ease of accessing participants. However, the focus in the group interviews remained on individual views and explanations, rather than adhering to traditional focus group methods and techniques [27]. 
A member of the research team (AT) carried out interviews, addressing the following topics, through a series of open ended questions exploring:

- Current stroke rehabilitation practice

- The potential role and use of visualisation technology within their current workplace environment

- The potential use and integration of the visualisation technology within their practice

The interview guides were developed by the study team with reference to the literature and discussion with stroke user groups who contributed to the early design and development of the visual tools and who discussed their personal experiences of stroke rehabilitation.

\section{Visualisation Technology}

Each of the three proposed trials employed a different visualisation technology, as described previously in table 1.

\section{Sample and Recruitment}

Key stakeholder clinicians from across the three envisage stroke trial sites, including those attending the pre-trial workshops, were approached by the leads for each of the trials and were asked if they would be willing to consent to a semi-structured interview with AT, to capture their views and perceptions about the visualisation technology. If they expressed an interest in participating, AT sent an email or contacted them individually by telephone to arrange a date and time to discuss the study, and if appropriate, to carry out the interview. Although all participants were able to provide an informed view about the visualisation technology, the sample was 
effectively one of convenience. All data collection was carried out in the clinical setting, and comprised a combination of individual and group interviews. The interviews lasted, on average, around forty five minutes (range thirty to sixty minutes). Verbal consent was given by all clinicians prior to the interview commencing and all interviews were audio-recorded and transcribed verbatim. The digital recorder failed during one interview, and consequently, notes were made by the researcher, AT, and were sent to the therapist for checking, following the interview.

\section{Data Analysis}

Framework analysis was used for the analysis of the interview data [28]. This approach is a robust and transparent analytic strategy, and is particularly useful for facilitating access to and understanding of the process of interpretation for those unfamiliar with qualitative data analysis. Framework analysis comprises five steps:

- Familiarisation: the analyst repeatedly reads the transcripts and starts to gain an overview of the data

- Identifying a thematic framework: Using processes of abstraction and conceptualisation, the analyst identifies key issues, concepts and themes. A thematic framework is constructed.

- Indexing: The thematic framework is systematically applied to all data.

- Charting: Data are reorganized and rearranged within the thematic framework.

- Mapping: The analyst identifies the key characteristics of the data, mapping and interpreting the data set as a whole. 
AT transcribed the interview recordings, and completed descriptive summary notes to provide information about the context and impressions of the interviews. AT led on the analysis, using the above steps to develop a thematic matrix inductively. She communicated frequently with other team members AM, DL and CB to discuss, develop and refine the theme matrices over the course of the project. Regular meetings with other team members from different disciplinary backgrounds ensured that tacit assumptions were surfaced and discussed.

\section{Rigour}

A number of strategies were employed to enhance the rigour or trustworthiness of the qualitative data generation [24]:

- The descriptive summaries noting additional information about the context for the interviews provided opportunity for further interrogation and reflection on the data

- $\quad$ AT shared successive iterations of the findings with AM and DL who questioned and commented on the developing thematic matrix. This practice supported a deeper awareness of potential meanings of the data and the implicit assumptions which AT brought to her interpretation, helping to surface and explore these

- The wider team, including CB, discussed successive accounts of the findings, exploring different ways of representing the themes to facilitate a rich and coherent interpretation 


\section{Findings}

Sixteen rehabilitation therapists participated in semi-structured interviews from across a range of disciplines involved in stroke rehabilitation: orthotists (two); physiotherapists (five) and occupational therapists (nine). Of these interviews, seven were carried out with individuals, with the remaining nine rehabilitation professionals being interviewed in three groups of two and one group of three, respectively. Their years of experience ranged from $2-26$ years, with the majority practicing in the NHS for ten years or more. Two of the participants worked in a specialist centre, an additional participant worked in a community hospital, and the remainder were from both acute and community rehabilitation teams. Participants were drawn from two NHS Health Boards in south central Scotland.

Three main themes emerged through analysis of the data:
a) Potential uses of the visualisation tools
b) Integration within current service provision
c) Trial evaluation involvement

This section will describe each of these, drawing on excerpts from the data to illustrate and illuminate each theme in turn.

\section{a) Potential uses of the visualisation tools}

In general, the therapists were enthusiastic about the potential application of the visualisation tools. This was most evident when discussing upper limb rehabilitation where the need for new tools and techniques was particularly welcomed, as this excerpt illustrates: 


\begin{abstract}
"it's been a long time really in upper limb rehab since there's been anything very different, so it's quite exciting, 'cause upper limb comes in as a priority and then it goes out, comes in, it goes out, but it's one of these things that, at the end of the day, ... at the end of all the rehab people want to be able to use their arm" (RTSG1)
\end{abstract}

Therapists made reference to the use of the 'same old equipment' which had been in use for many years, and indicated that they were keen to move forward with the use of new intervention tools.

One of the key ways in which the therapists envisaged that the visualisation tools might be used was to enhance communication. Many discussed the challenges they experience when conveying the complexities of rehabilitation tasks and described how many patients struggle to understand the concepts that they are trying to communicate. They suggested that people with stroke can be a challenging group of patients to work with as they can present with cognitive, communication and visual problems, in addition to physical and mobility impairments. This therapist alludes to the freedom which the visualisation tools would provide to elaborate and clarify:

\begin{abstract}
"when you're, you're trying to, to kind of re-train movements and things that you have to, you're talking about maybe four or five different things, and trying to, to create a picture in someone's head, [it] can be very difficult, so rather than have to create it yourself, that picture is then transferred to that visualisation so you can talk round it and explain it ..." (RT1CH)
\end{abstract}


Enhanced communication within rehabilitation was described as promoting many benefits, including patient motivation, understanding and engagement. However, not all of the interviewees were universally positive about the potential of the visualisation tools, as highlighted in this excerpt:

“... stroke patients, eh, they tend to be elderly so part of my worry is cognitively, and also technology, that age of patient group are, they're not 'techno-friendly'. They're also not at, they don't tend to be as forceful as younger people who are determined to rehabilitate themselves, they tend to be more receivers of rehab than, they're not used to being interactive with healthcare, it's just a generational thing. Em so I feel stroke patients, elderly stroke patients are probably not where this technology is best targeted really, em but I could be wrong about that..." (RT1SG)

Conversely, however, a senior therapist expressed the view that the visualisation tools could actually promote patient ownership of the rehabilitation process. This therapist was one of the few participants to highlight the opportunities for enhanced communication between the rehabilitation service providers and users, rather than simply communication from the therapist to the patient. The reference to the sharing of responsibility for successful rehabilitation is also therefore of particular interest within this context:

"the visualisations may help patients understand their rehabilitation tasks more and allow them to become more involved in their sessions with more communication occurring between the therapist and patient. The visualisations would help promote interaction and communication 
leading to the patient, hopefully, taking ownership of their rehabilitation tasks leading to them progressing positively through the programme." (RT1WH)

A second potential use for the visualisation tools was the capture and monitoring of progress within rehabilitation. For the therapists, this was generally expressed in terms of assessment, as illustrated in this excerpt:
"It would probably be a good assessment tool, looking at it because you, you don't have kind of bits of clothing, you don't have other things distracting you, you can look specifically at the alignment and the movement of a limb and it just makes it so much clearer......so you can, if you're then using that as an assessment tool and you can then, again pick away at the, the little problems and try and sort them" (RT1CH)

The ability of the tools to "unmask" and show both the patient and therapist the alignment and movement of the limb clearly was described as new and potentially very helpful. Therapists across each of the three trial scenarios consistently discussed how they "feel" the biomechanical position and alignment of the joints or instinctively know by seeing how the joint is moving. This could be challenging, and was acknowledged as subjective and potentially problematic, with the visualisation tools potentially providing more accurate and reliable information:

"... having the ability to view the movement of the upper limb during the session will allow the therapist to measure the level of efficiency of the movement, as currently this is a subjective measure (ie eye of the therapist). Currently there can be variations between therapists but by having the 
movement recorded, it provides an objective measure rather than the therapists needing to depend on the subjective clinical notes of the therapist" (RT1WH)

Some expressed the view that such tools would be of more use to less experienced colleagues:
"I think orthotists are very good at clinical biomechanics ... I think we are very good at being able to snapshot gait analysis in people and also do it multi- tasking while we are chatting with them. I am an experienced clinician, I think it's more difficult when you are a new clinician which is why I am interested in the visualisation, em because I'm maybe not the target audience for that 'cause in a sense l've done a lot so I'm used to this kind of patient group and the kinda problems" (RT1SG)

The opportunity for the visualisation tools to enable patients themselves to see progress within their rehabilitation was also described as an important benefit:
“... they'll [ie the patients] see the change - do you know it's a lot harder to see a change in yourself when you're doing an activity, whereas if they're actually seeing a change on the screen from their previous, 'cause you can show them their previous recordings, em and show them the difference" (RT2CH)

\begin{abstract}
As with the benefits of improved communication, the potential for patients to track their own progress was described as positively impacting on motivation: "I think it would really motivate them as well" $(\mathrm{RT} 2 \mathrm{HH})$; "(the tools would) encourage them to
\end{abstract}


keep with it, and to practice more and encourage them 'cause that's the difficulty we have, particularly in the community" (RT2WH)

One of the occupational therapists described a potential difficulty with patients making sense initially of the visualisations, but suggested that this could probably be overcome:

“... once you sort of adjust to the fact that 'Oh, there's not a head and oh, there's not a leg or foot', once you get past that and then you actually see what it's demonstrating, that it's very visual and it's clear, they (ie the patients) will probably adjust, 'cause you've not really seen anything like that before so its new, so once you get your head round that and you think 'Oh, it's actually not particularly relevant if there's a head or a leg, is there, cause we're not looking at that, that's not the focus ...'” (RT3HH)

The final potential benefit of the visualisation tools which the therapists described was as an education tool: to discuss clinical issues with other colleagues; to consider and plan future rehabilitation sessions; and to help educate junior members of the rehabilitation team, as this interviewee highlights:

\footnotetext{
"the use of the visualisations, in an educational way for novices, or even undergraduates or quite often my colleagues, who will specialise in other areas and very rarely see neuro ....... we tend to work in a way that we would tend to always point the patients at the right person, but then there are others where you're trying to mentor them or give them support where something like this might be useful as an educational tool 'cause its often difficult for me to articulate how I can do that" (RT1SG)
} 
The novelty and clinical benefits afforded by the visualisation tools were seen as being beneficial to both therapists and patients, as illustrated here:

“... I actually do think for both, it'll work for both us as therapists and the patients, it should show something to motivate both of us, to work together" (RT4WH)

\section{b) Integration within current service provision}

In contrast to the direct benefits for people with stroke and therapists within the therapeutic interaction described above, this theme captures the potential impact of the visualisation tools on service provision primarily from a service provider perspective.

Although generally positive about the potential use of the tools, the rehabilitation staff were very conscious of the practical constraints to adoption within current service configuration. Time was identified as a challenge, particularly within the context of rehabilitation for recovery of arm function following stroke:

“... you've got quite a lot of priorities in acute ... you've not just got upper limb, you know the, the big push is to get the ... em, the people up, get people balancing, get people looking after themselves, and get people out the door" (RT3HH)

It was felt that some environments might be better able to make use of the visualisation tools than others:

“... we're always gunna struggle in the acute, 'cause we struggle to get through the caseloads so probably more pressure on beds, time and staffing, 
it's a very fast pace so it's probably not going to be us. I think the Community teams, or Out-patient teams of Day Hospitals, I think they would be the kind of places that would really benefit from it (ie the visualisation tools) because they are the ones that would be treating people for two hours a week" (RT3HH)

Another practical consideration about which some of the interviewees expressed concern was the size of the equipment required to run the visualisation programmes, which was viewed as constraint within some settings:

“... sort of operationally, there's, there's, there is nowhere to put something like that round there. There's no, I don't have any therapy treatment areas around there ... now l've only got, um, a wee kitchen, a wee bedroom and a wee toilet ... um, so I, I don't really see where I could set up, there is no place round in the Day Hospital I could set up ... that type of equipment" (RT4WH)

The equipment size also potentially inhibited the tools' use within people's own homes:

'... the size of all the equipment, and it's a bit like big, and setting it up and it's not always feasible for a lot of the community ones (ie patients) to come to somewhere like the (Rehabilitation) Centre. A lot of people don't have transport and I think maybe in the Day Hospital there's transport already there, but for people in the home, I can see that there might be some difficulties" (RT1CH)

However, these difficulties were not seen as insurmountable, and some of the interviewees could envisage using the tools in the community with access to more 
software, or with modifications to enhance the portability of the equipment. Others could see the benefit of using the visualisation tools in the home environment, when the patient has been discharged from acute care:

"Yeah, it's not really until they're back on their feet that they (ie patients) take the time to think ... about other things ... I want to be able to do more ...." (RT3CH).

For guaranteed integration into routine clinical practice, one of the senior therapist interviewees provided a checklist of criteria which would need to be met:

"... the main features of the tool are that (it) is proven to be cost effective, portable, as most rehab is carried out in the community in people's homes and therefore the tool would need to be portable and easily plugged into a patient's television, for example. I am very positive about this tool and it's potential, especially in upper limb rehabilitation, as this is an area that tends to be neglected in acute care, as the focus is getting the patient to stand, then walk, enabling them to go home" (RT1WH)

\section{c) Trial evaluation involvement}

Another useful contribution of Normalisation Process Theory (previously highlighted in the Introduction section) is the explicit attention to how trial parameters can be optimised, thus assisting in the design of robust, feasible and meaningful randomised controlled trials [23]. As previously described in the Introduction, this initial study was carried out prior to three linked trials, evaluating the use of different visualisation tools with people with stroke within three different contexts. The 
qualitative data highlighted some issues which it would be useful to consider in future trials of visualisation equipment.

The participants in this study offered a variety of personal perspectives about their involvement in the research evaluation of the tools. As with the second theme above, this third and final theme was highly influenced by practical concerns arising from the daily working lives of the rehabilitation professionals, with lack of time being again identified as problematic:

"(I am) quite excited actually, just something different, but obviously when there's the, the concerns about the extra workload because .. time is the biggest issue for all of us just now, you're always aware you, you want to do something, but you, that while you're doing that something, what's happening to the work you should be doing?" (RT1WH)

\begin{abstract}
Although there was a sense of concern about 'juggling' both their clinical workloads and their involvement in trials, many wanted to be involved in research to help improve their knowledge and contribute to research evidence to support their clinical practice. However, many interviewees highlighted the importance of keeping all involved health professionals informed about the progress of trials, as many research studies just 'fall away' with no-one knowing the outcomes:
\end{abstract}

"It's good to know there is a project and I hope it ... doesn't fall by the wayside 'cause it looks really good ... other projects did get completed but there was no, never got any feedback about it, we didn't really get to find out what the outcome of the trial was and there's not anybody 
championing it, you know, for you to take it forward, so hopefully this one will be different" (RT3CH)

Several of the senior staff made a direct link between the requirement for sound evidence of effectiveness and increased funding for their service, as illustrated in this excerpt:

“... the only way I will get any more staffing is if I can persuade a service who's already got a tight budget to give me some of their money, because I can save them money in another way and we are getting better at doing that, but our evidence-based in shockingly poor. It's all consensus and low-grade, there's very, very few RCTs which is why I'm delighted we're doing this one" (RT1SG).

\section{Discussion}

To our knowledge, this is the first time in which the views and expectations of therapists involved in stroke rehabilitation about innovative visualisation technologies have been reported. Given the priority attached to improvements in arm function, and balance and mobility by stroke stakeholders [29], it is encouraging that, in general, the interviewees were enthusiastic about the potential for introducing these new interventions into their practice. Several participants also suggested that visualisation technology could facilitate better partnership working between the therapist and the person with stroke within stroke rehabilitation. A requirement to promote the active involvement of patients with stroke in rehabilitation has been highlighted in several clinical guidance documents [30] [31]. 
The findings suggest that the rehabilitation professionals in our study lack usable objective tools to measure the details of movements, supported to some extent by recent publications which call for further improvement and evaluation of some outcome measures in stroke [32] [33]. However, it would appear that more familiarity with the measures recommended within these publications would also be beneficial to complement participants' experiences of subjectively analysing movements. This is particularly true of early career professionals who don't have as large a base of experience to draw on for patients with unusually presenting movement problems. The participants in the study reported a lack of appropriate tools for use in upper limb therapy in particular, in comparison to lower limb therapy which seems to be given priority in order to improve patients' mobility.

One of the few reservations highlighted concerned the limitations of 'elderly stroke patients' to interact with the technology and thus benefit from it. This may in part reflect a rather negative stereotype of older peoples' capacity, and there is evidence to the contrary suggesting that, for example, older peoples' use of the internet is rapidly increasing [34]. However, a new publication suggests that sensory and cognitive impairments can limit the engagement of older people with technology [35]. Sallinen et al [35] recommend that older service users are involved in both the design of and guidance about the use of new technology. This suggests that some attention could usefully be given to how the visualisation tools within this study could be introduced to service users with stroke, and how their potential is explained.

To pre-empt concerns such as these occurring within our three 'envisage' stroke work streams, a user group of stroke survivors was pre-specified and established in the pre-trial phase to inform the design and development. As part of this, a 
technology showcase day was held in the laboratory at one of the Universities to discuss the technology and any concerns people may have had. Pre-trial focus groups were held with both stroke survivors and health professionals to get feedback on prototypes of the visualisations, and several changes were made to the design of the tools to incorporate this feedback.

Challenges within the NHS environment concerning integration of the new visualisation technologies into current service provision are captured within the second theme. Both (lack of) time and space were identified as potentially problematic, although the evidence to demonstrate that rehabilitation with the tools was cost effective could mitigate these constraints.

In this study, the focus was on the potential of the visual feedback, utilising the available motion capture technology at the time of the study. However, the motion capture technology used is interchangeable and can be improved as this is a rapidly developing area - becoming more accurate, smaller and easier to use. A balance needed to be found therefore between addressing the concerns of the therapists in regard to the practical time implications and the focus of the evaluation.

If viewed as a 'complex intervention', Normalisation Process Theory [20] [21] provides a framework for understanding how stroke rehabilitation using visualisation technologies can become 'normalised' or a part of routine practice. Our interviews suggest that the participating therapists understood and valued the purpose and potential benefits of the intervention ('coherence' and 'cognitive participation'). In general, they also perceived the visualisation technologies as contributing to their mission of effective stroke rehabilitation and appeared in theory prepared to work to facilitate these novel interventions ('collective action') 
NPT also highlights some potential strategies to enhance and optimise the execution of trials evaluating visual technologies. Whilst therapists demonstrated an appreciation of the need to evaluate the technology (demonstrating 'coherence'), their concerns suggest that for the trials to facilitate the normalising of the use of these tools within rehabilitation, issues around a perceived increased time burden should be explicitly addressed (impacting on 'cognitive participation' and 'cognitive action'). Interviewees suggested that lack of feedback regarding the progress and outcomes of previous trials could negatively influence their enthusiasm to champion novel interventions (ie impacting on 'collective action' and 'reflexive monitoring'). Regular contact, feedback and support would therefore assist the conduct of trials such as those within the envisage programme of work.

Our proposal that NPT has potential utility when considering the implementation of visualisation technologies is supported by UK Medical Research Council guidance [36]. Within this guidance, NPT is highlighted as a robust theory which can inform a process evaluation of complex interventions, through consideration of the impact of context on implementation of interventions.

Several limitations are evident within this study:

- Although of a reasonable size for a qualitative study with sixteen participants, our sample of rehabilitation professionals was one of convenience, rather than, for example, being purposively recruited. This has therefore limited the extent to which we could identify and explore questions arising during the course of interpretation regarding the homogeneity of rehabilitation staffs' views. For example, we have not explicitly investigated the influence of years' 
practice experience on staffs' views and expectations about the visualisation technologies.

- In working primarily with a wider research team with expertise primarily in trial methodology, it was deemed more useful to use a non-specific 'subtle realist' research approach [24] teamed with accessible and explicit analytic procedures (in adopting Framework Analysis), rather than, for example, locating the work in a specific qualitative methodology, such as grounded theory, or phenomenology. This has resulted in more pragmatic and applied research, at the expense of in-depth theoretical considerations.

- Whilst strategies were used to enhance the rigour of the data interpretation, it would have been useful for additional members of the research team to independently code one or more of the transcripts, to enable comparison of codes and promote discussion about the potential meanings of the data.

However, in spite of these perceived limitations, the study has yielded novel and potentially useful findings which could impact positively on the development, evaluation and implementation of visualisation technologies within stroke rehabilitation.

Future recommendations for research within this area include engagement with service users participating in stroke rehabilitation using the novel interventions described here, to investigate their understanding, views and expectations about these technologies.

The randomised controlled trials taking place following this exploratory work have been characterised as feasibility studies (ie to test the feasibility of conducting definitive trials to explore the effectiveness and cost effectiveness of the visualisation 
technologies). Prior to widescale promotion and adoption of the tools, it will be necessary to evaluate the interventions through larger definitive trials. These trials would also need to include refinements to the visualisation tools and motion capture technology based on the feedback from the feasibility studies. We also recommend including embedded qualitative studies to continue to explore the subjective dimensions impacting on the uptake and use of such interventions.

Whilst the use of Normalisation Process Theory in the evaluation of computer technologies in health has been limited, publications to date suggest that it provides a useful way to explore the work required in order to implement such technologies [37] [38]. At its most basic, the theory helps with a distinction between the technology, and 'a set of practices related to that technology' (Pope et al [38], pp1), both of which need consideration if the technology is to be successfully implemented. We believe that NPT has great potential to assist in designing, evaluating and implementing such health technologies. 


\section{Acknowledgements}

The research programme on which this work is based was funded through Lifelong Health and Wellbeing, a cross-research council initiative in partnership with the UK health departments and led by the Medical Research Council, grant ID: 91021.

The writing of the research paper was supported by the National Institute for Health Research (NIHR) Collaboration for Leadership in Applied Health Research and Care Wessex at University Hospital Southampton NHS Foundation Trust. The views expressed are those of the authors and not necessarily those of the NHS, the NIHR or the Department of Health.

\section{Declaration of Interest}

The authors report no declaration of interest. 


\section{References}

1. Durward B, Baer G and Rowe P (1997) Functional Human Movement: Measurement and Analysis. London: Butterworth Heinemann.

2. Macdonald AS, Loudon D and Docherty C (2009) Innovation in envisioning dynamic biomechanical data to inform healthcare and design guidelines and strategy. NDA Research Programme: University of Sheffield. Accessed on: 12 May 2015. Available at: http://www.newdynamics.group.shef.ac.uk/ndafindings-1.html

3. Craig P, Dieppe P, Macintyre S, Michie S, Nazareth I and Petticrew M (2008) Developing and evaluating complex interventions: new guidance. London: Medical Research Council.

4. Loudon D, Macdonald AS, Carse B, Thikey H, Jones L, Rowe PJ, Uzor S, Ayoade $\mathrm{M}$ and Baillie L. Developing visualisation software for rehabilitation: investigating the requirements of patients, therapists and the rehabilitation process. Health Informatics Journal 2012; 18: 171-180.

5. De Assis GA, Corrêa AGD, Martins MBR, Pedrozo WG and de Deus Lopes R. An augmented reality system for upper-limb post-stroke motor rehabilitation: a feasibility study. Disability and Rehabilitation: Assistive Technology 2014; 4: $1-8$.

6. Green $\mathrm{D}$ and Wilson $\mathrm{PH}$. Use of virtual reality in rehabilitation of movement in children with hemiplegia. Disability and Rehabilitation 2012; 34: 593-604.

7. Mirelman A, Bonato P, Deutsch JE. Effects of training with a robot-virtual reality system compared with a robot along on the gait of individuals after stroke. Stroke 2009; 40; 169-74. 
8. Sampson M, Shau Y-W and King MJ. Bilateral upper limb trainer with virtual reality for post-stroke rehabilitation: case series report. Disability and Rehabilitation: Assistive Technology 2012; 7: 55-62.

9. Yang Y-R, Tsai M-P, Chuang T-Y, Sung W-H and Want R-Y. Virtual-reality based training improves community ambulation in individuals with stroke: $A$ randomized controlled trial. Gait and Posture 2008; 28: 201-6.

10. World Health Organisation (2001). International classification of functioning, disability and health. Geneva: World Health Organisation.

11. Lohse KR, Hilderman GE, Cheung KL, Tatla S and Van der Loos MHF. Virtual reality therapy for adults post-stroke: a systematic review and metaanalysis exploring virtual environments and commercial games in therapy. PLoS One 2014; 9; e93318. doi: 10.1371/journal.pone.0093318.

12. Rosewilliam S, Roskell CA and Pandyan AD. A systematic review and synthesis of the quantitative and qualitative evidence behind patient-centred goal setting in stroke rehabilitation. Clinical Rehabilitation 2011; 25: 501-14.

13. Nicholson SL, Donaghy M, Johnston M, Sniehotta FF, van Wijck F, Johnston D, Greig C, McMurdo ME and Mead G. A qualitative theory guided analysis of stroke survivors' perceived barriers and facilitators to physical activity. Disability and Rehabilitation 2014; 36: 1857-68.

14. Levack WMM, Dean SG, Siegert RJ and McPherson KM. Navigating patientcentred goal setting in inpatient stroke rehabilitation: how clinicians control the process to meet perceived professional responsibilities. Patient Education and Counseling 2011; 85: 206-13.

15. Drabble SJ, O'Cathain A, Thomas, Rudolph A and Hewison J. Describing qualitative research undertaken with randomized controlled trials in grant 
proposals: a documentary analysis. BMC Medical Research Methodology 2014; 14: doi:10.1186/1471-2288-14-24.

16. O'Cathain A, Thomas KJ, Drabble SJ, Rudolph A and Hewison J. What can qualitative research do for randomised controlled trials? A systematic mapping review. BMJ Open 2013; 3: doi:10.1136/bmjopen-2013-002889.

17. Mawson S, Nasr N, Parker J, Zheng H, Davies R and Mountain G. Developing a personalized self-management system for post stroke rehabilitation; utilizing a user-centred design methodology. Disability and Rehabilitation 2014; 9: 521-8.

18. Parker J, Mawson S, Mountain G, Nasr N, Davies R and Zheng H. The provision of feedback through computer-based technology to promote selfmanaged post-stroke rehabilitation in the home. Disability and Rehabilitation: Assistive Technology 2014; 9: 529-538.

19. Lindqvist $\mathrm{E}$ and Borell L. Computer-based assistive technology and changes in daily living after stroke. Disability and Rehabilitation: Assistive Technology 2012; 7: 364-71.

20. May CR, Mair F, Finch T, MacFarlane A, Dowrick C, Treweek S, Rapley T, Ballini L, Ong BN, Rogers A, Murray E, Elwyn G, Légaré F, Gunn J and Montori VM. Development of a theory of implementation and integration: Normalization Process Theory. Implementation Science 2009; 4: doi:10.1186/1748-5908-4-29.

21. May C and Finch T. Implementing, embedding, and integrating practices: An outline of Normalization Process Theory. Sociology 2009; 43: 535-554.

22. May, C., Murray, E., Finch, T., Mair, F., Treweek, S., Ballini, L., Macfarlane, A. and Rapley, T. (2010) Normalization Process Theory On-line Users' Manual 
and Toolkit. Available from http://www.normalizationprocess.org [Accessed on 21st August 2015].

23. Murray E, Treweek S, Pope C, MacFarlane A, Ballini L, Dowrick C, Finch T, Kennedy A, Mair F, O'Donnell C, Ong BE, Rapley T, Rogers A and May C. Normalisation process theory: a framework for developing, evaluating and implementing complex interventions. BMC Medicine 2010; 8: doi:10.1186/1741-7015-8-63.

24. Ballinger C. Writing up rigour: Representing and evaluating good scholarship in qualitative research. British Journal of Occupational Therapy 2004; 67: 540-6.

25. Burns Cunningham K. Social research design: framework for integrating philosophical and practical elements. Nurse Researcher 2014; 22: 32-37. 26. Mason J (2002) Qualitative Researching ( $2^{\text {nd }}$ edn) London: Sage Publications.

27. Morgan DL (1997) Focus Groups as Qualitative Research. London: Sage. 28. Richie J and Spencer L. Qualitative data analysis for applied policy research. In Bryman A and Burgess RG, eds., Analysing Qualitative Data, London: Routledge; 1994. p173-194.

29. Pollock A, St George B, Fenton M and Firkins L. Top 10 research priorities relating to life after stroke-consensus from stroke survivors, caregivers and health care professionals. International Journal of Stroke 2014; 9; 313-20.

30. National Clinical Guideline Centre (2013). Stroke Rehabilitation Long term rehabilitation after stroke Clinical guideline 162. London: National Institute for Health and Care Excellence. 
31. Scottish Intercollegiate Guidelines Network (2010). Management of Patients with Stroke: Rehabilitation, Prevention and Management of Complications, and Discharge Planning A National Clinical Guideline No 118.

32. Alt Murphy M, Resteghini C, Feys $P$ and Lamers I. An overview of systematic reviews on upper extremity outcome measures after stroke. BMC Neurology 2015: 15; DOI 10.1186/s12883-015-0292-6.

33. Salter K, Campbell N, Richardson M, Mehta S, Jutai J, Zettler L, Moses M, McClure A, Mays R, Foley N and Teasell R. (2013) Ch. 21. Outcome Measures in Stroke Rehabilitation. In Evidence Reviews R Teasell, M Richardson, L Allen, N Hussein (eds) Evidence-Based Review of Stroke Rehabilitation. Available at: www.ebrsr.com/evidence-review/21-outcomemeasures. Accessed on: 21 August 2015).

34. Brooks C, Ballinger C, Nutbeam D and Adams J. Literacy levels required to understand regularly accessed falls prevention websites aimed at the public. Journal of Physical Therapy and Health Promotion 2013; 1: 8-14.

35. Sallinen M, Hentonen $\mathrm{O}$ and Kärki A. Techology and active agency of older adults living in service house technology. Disability and Rehabilitation: Assistive Technology 2015; 10: 27-31

36. Moore G, Audrey S, Barker M, Bond L, Bonell C, Hardeman W, Moore L, O'Cathain A, Tinati T, Wight D and Baird J. (2014) Process Evaluation of Complex Intervention UK Medical Research Council (MRC) Guidance. London: MRC Population Health Science Research Network.

37. May CR, Harrison R, Finch T, MacFarlane A, Mair FS and Wallace P. Understanding the normalization of telemedicine services through qualitative 
evaluation. Journal of the American Medical Informatics Association 2003; 10 :596-604.

38. Pope C, Halford S, Turnbull J, Prichard J, Calestani M and May C. Using computer decision support systems in NHS emergency and urgent care: ethnographic study using normalisation process theory. BMC Health Services Research 2013; 13: doi:10.1186/1472-6963-13-111. 
Table 1. Description of envisage stroke trials and visualisation technologies being evaluated

\begin{tabular}{|c|c|c|}
\hline Envisage Stroke Trial Workpackage & Visualisation technology evaluated & Example of visualisation \\
\hline $\begin{array}{l}\text { WP4 Lower limb stroke rehabilitation for } \\
\text { patients with recent stroke, within the context } \\
\text { of community rehabilitation carried out with a } \\
\text { therapist }\end{array}$ & $\begin{array}{l}\text { Motion capture: Optitrack } \\
\text { Feedback: real-time i.e. the participant } \\
\text { performed their rehabilitation exercises while } \\
\text { viewing the screen } \\
\text { Visuals: virtual targets for specific exercises }\end{array}$ & \\
\hline $\begin{array}{l}\text { WP4a Upper limb stroke rehabilitation for } \\
\text { patients with recent stroke, within the context } \\
\text { of community rehabilitation with a therapist }\end{array}$ & $\begin{array}{l}\text { Motion capture: Polhemus } \\
\text { Feedback: real-time i.e. the participant } \\
\text { performed their rehabilitation exercises while } \\
\text { viewing the screen } \\
\text { Visuals: 3D view enabling different viewpoints } \\
\text { to highlight compensatory movements }\end{array}$ & \\
\hline $\begin{array}{l}\text { WP5 Diagnosis and fitting of ankle foot } \\
\text { orthoses (AFOs) with patients with stroke, } \\
\text { within a laboratory setting, involving a multi- } \\
\text { disciplinary team }\end{array}$ & $\begin{array}{l}\text { Motion capture: VICON } \\
\text { Force measurement: Kistler force plates } \\
\text { Feedback: offline i.e. a participant's walk was } \\
\text { recorded, then the person would sit down and } \\
\text { view their data on screen } \\
\text { Visuals: visual representation of data to show } \\
\text { how the AFO has modified the person's gait }\end{array}$ & 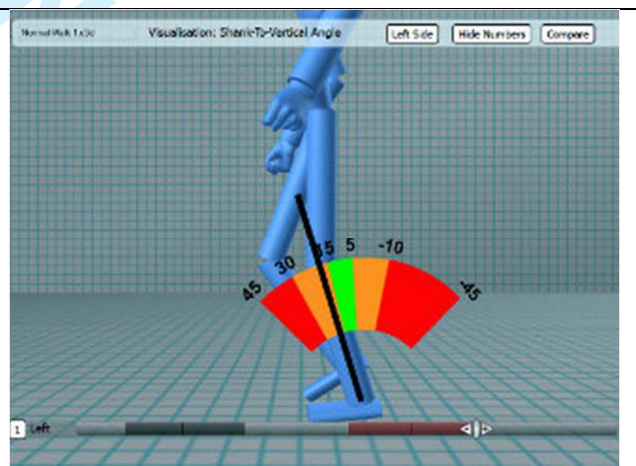 \\
\hline
\end{tabular}


Table 2. The four core constructs of Normalisation Process Theory [22]

\begin{tabular}{|c|c|}
\hline Core construct and definition & Example \\
\hline $\begin{array}{l}\text { Coherence: } \\
\text { The sense-making work that people } \\
\text { individually and collectively undertake } \\
\text { when operationalising new/revised } \\
\text { practices }\end{array}$ & $\begin{array}{l}\text { In carrying out a randomised controlled } \\
\text { trial (RCT) in a clinical setting, } \\
\text { coherence is required in order to } \\
\text { distinguish and differentiate between } \\
\text { work required to carry out the trial, and } \\
\text { work required in routine clinical practice. }\end{array}$ \\
\hline $\begin{array}{l}\text { Cognitive participation: } \\
\text { This refers to the relational work which } \\
\text { people need to do to support and } \\
\text { sustain the new/revised practice }\end{array}$ & $\begin{array}{l}\text { In executing an RCT on a ward setting, } \\
\text { it may be necessary for key stakeholder } \\
\text { clinicians to identify themselves, create } \\
\text { space and time to work together and } \\
\text { decide how to drive trial recruitment } \\
\text { forward. }\end{array}$ \\
\hline $\begin{array}{l}\text { Collective action: } \\
\text { This is the operational work that people } \\
\text { are required to carry out in order to } \\
\text { normalise the practice }\end{array}$ & $\begin{array}{l}\text { Clinical staff require particular relations, } \\
\text { knowledge, skills and resources to } \\
\text { successfully 'host' an RCT within their } \\
\text { clinical setting. }\end{array}$ \\
\hline $\begin{array}{l}\text { Reflexive monitoring: } \\
\text { The appraisal work which people do to } \\
\text { understand how the new/revised } \\
\text { practices impact them and others }\end{array}$ & $\begin{array}{l}\text { A clinical team may engage in reflexive } \\
\text { monitoring to evaluate the cost/benefits } \\
\text { to the patients, themselves and the } \\
\text { ward of participating in an } \mathrm{RCT} \text {, before } \\
\text { accepting another invitation to do so. }\end{array}$ \\
\hline
\end{tabular}




\section{IMPLICATIONS FOR REHABILITATION}

- There is little research exploring the use of visual software technologies featuring biomechanical data within stroke rehabilitation.

- The perspectives of stroke rehabilitation therapists about the potential of such tools are useful both in terms of planning trial evaluations, and implementation.

- Therapists were generally positive about the contribution of visual software tools in stroke rehabilitation, but highlighted a number of practical constraints which required addressing.

- Normalisation process theory provides a useful process evaluation methodology which can support both trial evaluation and implementation of such novel technologies within stroke rehabilitation. 